\section{Contrôle du trafic intracellulaire de Elk-1 par SUMO}

Sara Salinas, Robert A. Hipskind
Institut de Génétique moléculaire de Montpellier, CNRS, UMR 5535; IFR 122, 1919 , route de Mende, 34293 Montpellier Cedex 5, France.

robert.hipskind@igmm.cnrs.fr famille TCF (ternary complex factor) sont des acteurs majeurs de l'activation rapide du programme transcriptionnel déclenché en réponse à divers signaux physiologiques [1]. L'activité des trois membres de la famille TCF, Elk-1, SAP-1 et SAP-2/NET/ERP, dépend de leur état de phosphorylation, contrôlé par les mitogen activated protein kinases (MAPK) qui, à la suite de leur induction, sont responsables de la transmission du signal au sein de la cellule et, plus particulièrement, vers le noyau. Les TCF fonctionnent principalement dans le contexte d'un complexe ternaire, dans la plupart des cas avec le facteur de réponse au sérum (SRF, serum response factor) et l'élément de réponse au sérum (SRE, serum response element) situés dans les séquences régulatrices des gènes de réponse précoce [1]. Ce complexe semble être présent dans les promoteurs de façon constitutive in vivo, où le composant TCF, directement phosphorylé par une MAPK, est responsable de l'activation transcriptionnelle très rapide de ces gènes [1].

Récemment, Elk-1 a été décrit comme étant soumis à un deuxième type de modification post-traductionnelle: la conjugaison de SUMO (small ubiquitin-like modifier) au domaine de répression transcriptionnelle [2, 3]. La modification des protéines par SUMO semble avoir plusieurs rôles: elle peut régler certaines interactions protéine-protéine, l'import/export nucléaire, la localisation intracellulaire et la transcription [4]. Dans le cas d'Elk-1, modification par SUMO

semble nécessaire pour le

recrutement de l'histone désacétylase HDAC-2, enzyme qui réprimerait l'expression des gènes cibles par la désacétylation de la chromatine associée [5]. La phosphorylation activatrice d'Elk-1 mènerait, d'une part, à sa « déSUMOylation » et donc à la perte de liaison de I'HDAC-2 et, d'autre part, au recrutement du co-actition de SUMO (flèche rouge épaisse). vateur CBP ou p300 [6, 7]. Ce mécanisme pourrait expliquer plusieurs cas de répression de facteurs de transcription liés à leur état de «SUMOylation » [4].

En accord avec son rôle de régulateur transcriptionnel, $\varepsilon l k-1$ est localisé dans le noyau des cellules en culture. Deux régions de la protéine contrôlent cette localisation: la région comprise entre les acides aminés 55 et 83 , dans la partie carboxyterminale du domaine de liaison à l'ADN de la famille ETS, et la région comprise entre les acides aminés 137 et $157[8,9]$.

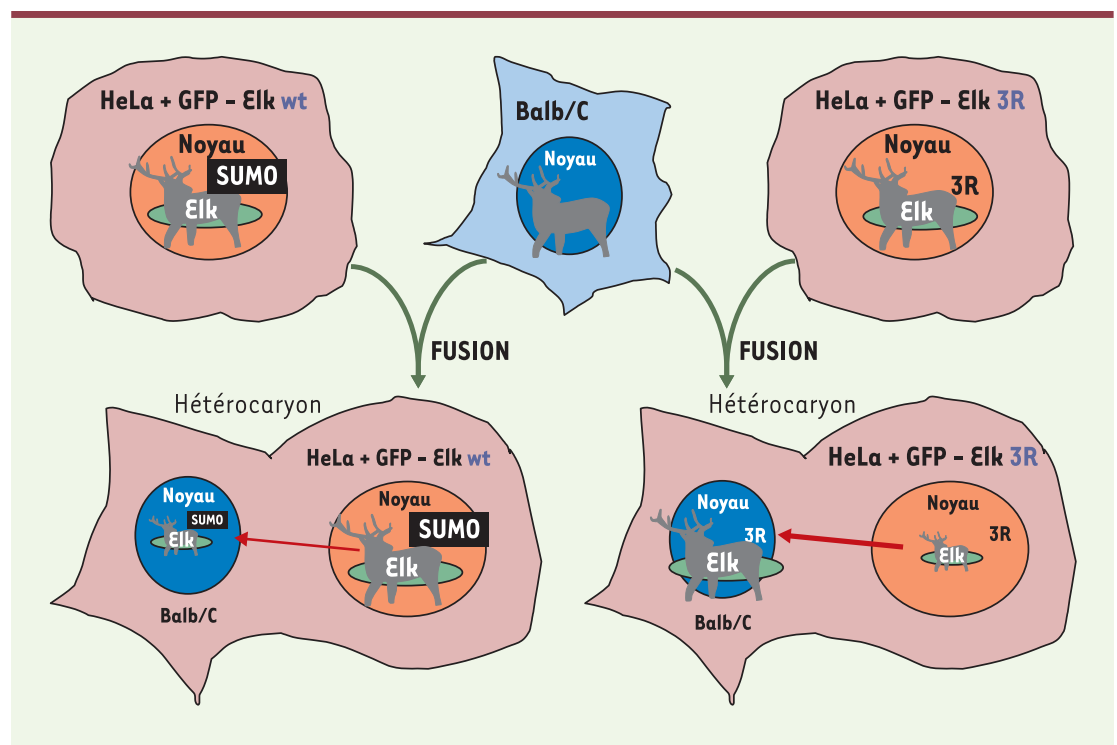

Figure 1. La conjugaison de SUMO empêche $\varepsilon_{l k-1}$ de faire la navette entre le noyau et le cytoplasme dans les hétérocaryons HeLa-Balb/C. La lignée cellulaire humaine HeLa co-exprime de façon ectopique SUMO et le facteur de transcription Elk-l étiqueté avec un marqueur de fluorescence vert (GFP, green fluorescent protein), soit sous sa forme sauvage (wt), soit sous une forme mutée dans les trois sites de conjugaison de SUMO (3R). Les cellules HeLa (en rose) sont ensuite fusionnées avec les cellules Balb/C de souris (en bleu) à l'aide de polyéthylène glycol. Dans les hétérokaryons, la forme sauvage de GFP-Elk-l est «SUMOylée » et transloquée vers le noyau des cellules Balb/C nettement plus lentement (flèche rouge fine) que le mutant $3 R$, insensible à l'ac- 
Récemment, nous avons montré, en exploitant la technique d'hétérocaryon, qu'عlk-l fait la navette entre le noyau et le cytoplasme. En fusionnant les cellules HeLa exprimant une version d'Elk-1 fluorescente avec des fibroblastes murins, nous avons observé une quantité croissante de GFP-Elk-1 dans les noyaux des cellules murines en fonction du temps après la fusion [3]. Ces observations traduisent le fait que la protéine $\varepsilon l k-1$ est exportée du noyau mais est également rapidement ré-importée, soit dans le noyau d'origine, soit dans le noyau résultant de la fusion cellulaire. De façon intéressante, un mutant d'عlk-1, incapable d'être conjugué à SUMO, fait la navette plus rapidement que la forme sauvage d'Elk-1, et la surexpression stable de SUMO dans les cellules HeLa aboutit à une diminution de la cinétique d'import/export d'Elk-l sauvage, sans avoir d'effet sur celle de la forme mutante d'Elk-1 (Figure 1). II semble donc que la conjugaison de SUMO à Elk-1 contrôle sa rétention nucléaire et/ou la cinétique de son import/export.

Afin de confirmer l'effet de SUMO sur la localisation d'Elk-l dans un contexte plus physiologique, nous avons fait appel aux études du groupe de J. Caboche, qui ont montré qu'દlk-1 est localisé à la fois dans le noyau et dans le cytoplasme des neurones du système nerveux central $[9,10]$. Cette localisation cytoplasmique est corrélée à la présence d'une isoforme neuronale d'Elk-l qui résulte d'un démarrage interne de la traduction à partir de la méthionine 55 , au milieu du domaine ETS [9]. L'expression de cette isoforme est induite par un traitement des cellules PC12 avec le nerve growth factor (NGF), qui entraîne, d'une part, une relocalisation cytoplasmique d'Elk-1 sauvage et, d'autre part, la croissance neuritique (Figure 2), un effet inhibé par le blocage de l'export nucléaire d'Elk-l sauvage [9]. La relocalisation cytoplasmique d'Elk-1 semble donc être importante pour le processus de différenciation. Dans ce contexte, nous avons testé l'ef- fet de l'état de SUMOylation d'Elk-l sur la différenciation des cellules PC12 sans induction par le NGF. L'expression du mutant «non-SUMOylable» d'Elk-1 induit l'extension des neurites plus fortement que la forme sauvage d'Elk-l [3]. La co-expression de SUMO inhibe la différenciation neuronale induite par la forme sauvage d'Elk-1 mais n'a aucun effet sur la forme «non-SUMOylable» d'Elk-1 (Figure 2). En revanche, en présence d'une forme dominante négative de l'enzyme de conjugaison UBC9, qui bloque le processus de «SUMOylation» endogène, Elk-l sauvage et mutant induisent la croissance neuritique de façon similaire. En résumé, on peut faire une parfaite corrélation entre la rétention d'Elk-1 dans le noyau contrôlée par SUMO et l'inhibition de la différenciation neuronale. Ces observations renforcent à la fois le modèle de P. Vanhoutte et al. [9] et l'importance de la conjugaison de
SUMO à Elk-l dans sa localisation nucléaire.

Ces résultats ajoutent $\varepsilon l k-1$ à la liste des protéines pour lesquelles la localisation nucléaire est contrôlée par SUMO et également aux protéines nucléaires faisant une navette dynamique entre le noyau et le cytoplasme [4]. Néanmoins, la difficulté de détecter Elk-1 dans le cytoplasme en dehors des neurones indique que cette dynamique penche fortement vers le noyau, permettant à Elk-l d'effectuer sa fonction de régulateur des gènes de réponse précoce. De plus, la «SUMOylation» d'Elk-1 pourrait avoir un deuxième rôle, celui d'assurer la répression des gènes cibles d'Elk-l en attendant un signal activateur. Les études à venir devraient permettre d'évaluer l'importance de cette modification vis-à-vis de la phosphorylation dans le fonctionnement d'Elk-1. $\diamond$

SUMO pins $\varepsilon \mid k-1$ in the nucleus

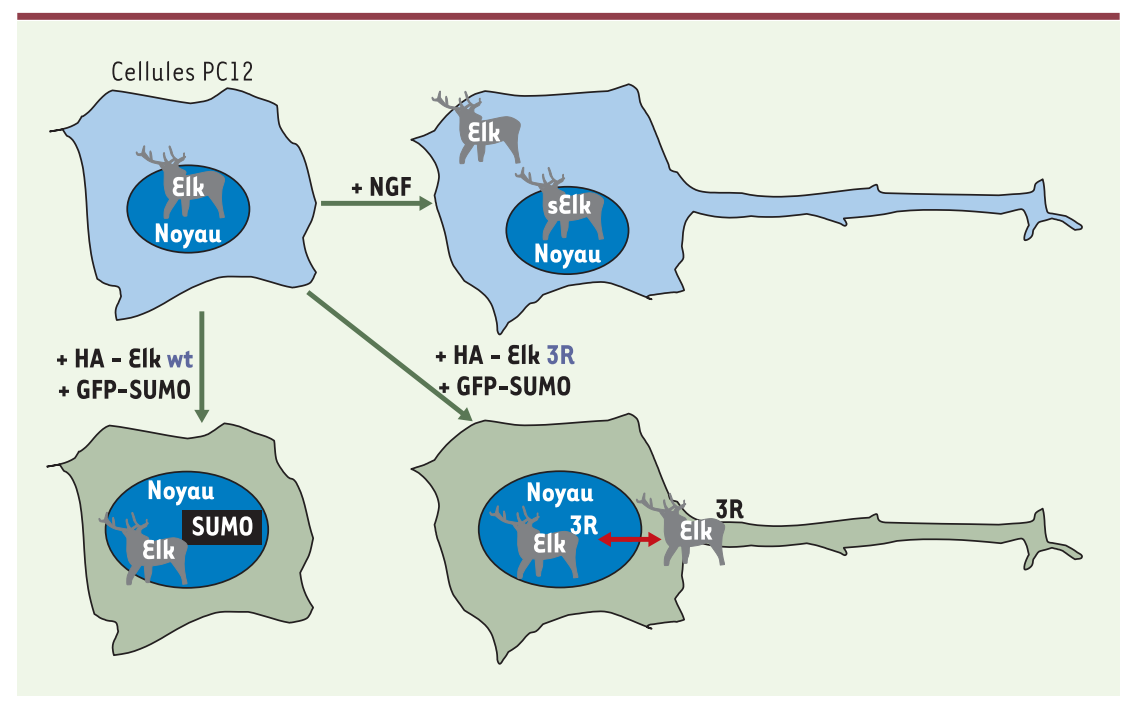

Figure 2. Le mutant « non-SUMOylable » d'Elk-1 peut induire la différenciation neuritique des cellules PC12. Les cellules PC12 en prolifération expriment la forme sauvage d'Elk-1 qui est localisée dans le noyau. Le nerve growth factor (NGF), qui induit la différenciation neuronale des cellules $\mathrm{PC12}$, stimule la synthèse d'une petite isoforme d'Elk-1 (sદlk) qui permet la localisation cytoplasmique d'Elk-l sauvage. La surexpression d'દlk-l sauvage - étiqueté par l'épitope hémagglutinine (HA-Elkwt) - avec SUMO - étiqueté avec un marqueur de fluorescence vert (GFP, green fluorescent protein) - n'a aucun effet sur la différenciation des cellules $\mathrm{PC} 12$. En revanche, la surexpression du mutant 3R d'Elk, qui est insensible à l'action de GFP-SUMO, stimule de façon significative la croissance neuritique des cellules $P C 12$. Ces observations suggèrent que la rétention d'Elk-l dans le noyau - dépendant de la conjugaison par SUMO - est associée à une inhibition de la croissance neuritique. 


\section{RéFÉRENCES}

1. Wasylyk B, Hagman J, Gutierrez-Hartmann A. Ets transcription factors: nuclear effectors of the RasMAP-kinase signaling pathway. Trends Biochem Sci 1998; 23: 213-6.

2. Yang $S H$, Jaffray $\varepsilon$, Hay RT, Sharrocks $A D$. Dynamic interplay of the SUMO and ERK pathways in regulating Elk-1 transcriptional activity. Mol Cell 2003; 12: $63-74$

3. Salinas S, Briancon-Marjollet A, Bossis G, et al. SUMOylation regulates nucleo-cytoplasmic shuttling of Elk-1. J Cell Biol 2004; 165: 767-73.
4. Seeler JS, Dejean A. Nuclear and unclear functions of SUMO. Nat Rev Mol Cell Biol 2003; 4: 690-9.

5. Yang SH, Sharrocks AD. SUMO promotes HDACmediated transcriptional repression. Cell 2004 ; 13: 611-7.

6. Janknecht R, Nordheim, A. MAP kinase-dependent transcriptional coactivation by $\varepsilon l k-1$ and its cofactor CBP. Biochem Biophys Res Commun 1996; 228: 831-7.

7. Li Q, Yang SH, Maeda Y, et al. MAP kinase phosphorylation-dependent activation of $\varepsilon l k-1$ leads to activation of the co-activator p300. EMBO J 2003; 22: 281-91.
NOUVELLE

\section{Polymorphismes de Plasmodium vivax Comment les interpréter? Quelles incidences en santé publique aujourd'hui ?}

Dominique Labie
8. Janknecht R, Zinck R, Ernst WH, Nordheim A. Functional dissection of the transcription factor Elk-1. Oncogene 1994; 9: 1273-8.

9. Vanhoutte P, Nissen J, Brugg B, et al. Opposing roles of $\varepsilon l k-1$ and its brain-specific isoform, short $\varepsilon l k-1$, in nerve growth factor-induced $\mathrm{PCl} 2$ differentiation. J Biol Chem 2001: 276: 5189-96.

10. Sgambato V, Vanhoutte $P$, Pages $C$, et al. In vivo expression and regulation of $\varepsilon l k-1$, a target of the extracellular signal-regulated kinase signaling pathway, in the adult rat brain. J Neurosci 1998; 18: 214-26.
Inserm U.567, Institut

Cochin, 24, rue du Faubourg

Saint-Jacques, 75014 Paris, France.

labie@cochin.inserm.fr
$>$ La fréquence du paludisme a au moins doublé au cours des trente dernières années. Touchant actuellement près de 500 millions de personnes par an, le paludisme est responsable de plus d'un million de décès, majoritairement en Afrique. L'explication en est sans doute l'interférence de plusieurs facteurs: résistance des parasites aux médicaments utilisés, résistance des moustiques vecteurs aux insecticides, changements climatiques, mais aussi facteurs sociaux tels que l'instabilité politique, la carence des services de santé et la pauvreté [1]. L'urgence du problème explique sans doute la multiplicité des abords, au premier chef desquels la recherche d'un vaccin. Une majorité de travaux ont été consacrés au Plasmodium falciparum, le seul massivement létal. Le Plasmodium vivax reste, cependant, le parasite dont la répartition est la plus étendue, responsable de 70 à 80 millions de cas morbides chaque année. II est responsable, en très large part, de la morbidité du paludisme en Asie centrale, du Sud et du Sud-Est, en Amérique latine et au Moyen-Orient. En Afrique, il n'est observé que dans les régions orientales ou du sud. Sa presque inexistence en Afrique occidentale et centrale s'explique par l'absence de l'antigène Duffy, récepteur érythrocytaire normal des protéines de surface du mérozoïte de $P$. vivax. Ces données épidémiologiques et la fréquence accrue des déplacements font aussi craindre sa réémergence dans des régions d'où le paludisme a été éradiqué, mais où le vecteur potentiel de sa transmission existe toujours. Les conséquences, bien que moins immédiatement dramatiques, sont importantes, tant pour la politique de santé qu'au niveau économique. Un obstacle majeur aux travaux de recherche est encore l'absence d'un système de culture en continuité du parasite et la nécessité d'entretenir les souches dans les érythrocytes de singes [2]. Un projet de séquence du génome, à l'instar de ce qui a été fait pour le $P$. falciparum, est en cours, mais n'a encore que des résultats partiels [3].
C'est à partir de séquences identifiées que différentes équipes ont entrepris la recherche de polymorphismes sur des souches de $P$. vivax originaires de différentes régions dans le monde. Ce type de travail a un double intérêt. II permet d'étayer les hypothèses concernant l'évolution du parasite et la transmission éventuelle d'un continent à un autre. II est aussi indispensable dans la recherche de séquences codant pour des protéines de surface qui seraient éventuellement les cibles d'une approche vaccinale. Les résultats sont encore fragmentaires, ils nécessitent d'être comparés entre eux car ils peuvent, en effet, paraître discordants. Un premier travail, en 2000, mettait en évidence le polymorphisme de deux antigènes exprimés en surface [4]. En 2002, une série de travaux portait à nouveau sur le gène codant pour la protéine de surface $1 \mathrm{du}$ mérozoïte, $m s p-1$, Ces travaux ont été menés, notamment, en Italie à l'occasion d'un cas de paludisme apparemment autochtone observé en Toscane 